Luciane Frizo Mendes

Selma Lancman ${ }^{2}$

\section{Reabilitação de pacientes com LER/DORT: contribuições da fisioterapia em grupo*}

\author{
Rehabilitation of patients with RSI/WRMD - the contribution of \\ group physical therapy
}

${ }^{1}$ Docente do Curso de Fisioterapia da Universidade Metodista de São Paulo -SP, Brasil.

${ }^{2}$ Docente do Departamento de Fisioterapia, Fonoaudiologia e Terapia Ocupacional da Faculdade de Medicina da Universidade de São Paulo - SP, Brasil.

*Artigo baseado em tese para obtenção de título de doutor de Luciane Frizo Mendes, intitulada A contribuição da fisioterapia em grupo na recuperação e reabilitação de pacientes com LER/DORT, defendida na Faculdade de Medicina, Universidade de São Paulo, em 2008.

\section{Contato:}

Luciane Frizo Mendes

Rua Ribeiro do Amaral, 585 - apto. 106.

CEP: 04268-000, São Paulo, SP.

E-mail:

lucianefrizo@ig.com.br
Recebido: 30/06/2009

Revisado: 18/03/2010

Aprovado: 23/03/2010

\section{Resumo}

Objetivo: Avaliar os benefícios da associação do tratamento cinesioterapêutico com as dinâmicas de grupo, numa abordagem mais integral do processo saúde-doença, na recuperação e na reabilitação de portadores de LER/DORT em relação à funcionalidade e ao retorno ao trabalho. Método: vinte e quatro pacientes diagnosticados com LER/DORT foram distribuídos aleatoriamente em intervenções individuais e grupais, em 2008. O protocolo de cinesioterapia foi o mesmo nas duas intervenções e durou 10 sessões. Após os exercícios, foram abordados aspectos psicossociais importantes para o tratamento. A análise das intervenções ocorreu através da avaliação da funcionalidade pelo Questionário DASH e por uma entrevista semiestruturada para avaliar qualitativamente o impacto dessas intervenções no quadro clínico e na qualidade de vida após o tratamento. Resultados: A avaliação da funcionalidade identificou que em nenhuma das intervenções houve alteração das funções dos membros superiores. Na análise das entrevistas, observou-se que os participantes relataram uma percepção de melhora do quadro clínico e da funcionalidade em suas vidas, mas que não foi suficiente para assegurar o retorno ao trabalho. Conclusão: As reflexões criadas nas duas intervenções permitiram uma abordagem mais global do processo de adoecimento, recuperação e reabilitação do paciente com LER/DORT, mas não foram suficientes para garantir o retorno ao trabalho.

Palavras-chave: saúde do trabalhador; transtornos traumáticos cumulativos/ reabilitação; modalidades de fisioterapia; grupos.

\section{Abstract}

Objective: Assess the benefits of associating kinesiotherapeutic treatment to group dynamics in a more integrative approach for the health-illness process of recovering and rehabilitating RSI/WRMD patients, regarding their functionality and return to work. Method: In 2008, 24 RSI/WRMD patients were randomly distributed for individual and group interventions. The kinesiotherapeutic protocol was the same for both interventions and took 10 sessions. After the exercises, psychosocial aspects considered important to the treatment of RSI/WRMD patients were addressed individually and in groups. The impact of the interventions on patients' clinical condition and on their quality of life was evaluated by using DASH Questionnaire for patients' functionality and semi-structured interviews. Results: The assessment of patients' upper-limb functionality revealed that there was no change in their functional state in none of interventions. The interviews, on the other hand, indicated that the participants improved their clinical condition and functionality, but this improvement was not enough to assure their return to work. Conclusion: The possibility of reflection during both types of intervention allowed a more comprehensive approach to deal with RSI/WRMD patients' illness, recovery and rehabilitation processes, but it was not enough to ensure their return to work.

Keywords: occupational health; cumulative trauma disorders/rehabilitation; modalities of physical therapy; groups. 


\section{Introdução}

As ações de saúde do trabalhador na rede pública de serviços de saúde estavam inseridas, até a década de 1980, nos Programas de Saúde do Trabalhador. Com a institucionalização do SUS (Sistema Único de Saúde), foram reorganizadas e integradas em Centros de Referência, tornado-se uma estrutura de apoio na rede básica para os problemas de saúde do trabalhador. Esses Centros garantem desde a assistência às ações até atividades em vigilância nas empresas, privilegiando tanto a prevenção de agravos e promoção da saúde, quanto a assistência à saúde dos trabalhadores acometidos por doenças relacionadas ao trabalho (BRASIL, 2001).

A Lesão por Esforço Repetitivo ou Distúrbio Osteomuscular Relacionado ao Trabalho (LER/DORT) é uma das mais importantes causas de afastamento e destaca-se entre as maiores repercussões na saúde do trabalhador decorrentes das transformações do trabalho, principalmente dos novos modelos organizacionais e de gestão (BRASIL, 2001).

Os serviços de referência em saúde do trabalhador de diversas regiões do país recebem uma alta demanda de trabalhadores portadores de LER/DORT, que buscam associar legalmente o diagnóstico com o trabalho, orientações previdenciárias e recursos terapêuticos (SETTIMI et al., 1998).

O tratamento desses trabalhadores não deve considerar apenas aspectos clínicos e deve incluir também uma preparação para o retorno ao trabalho, algumas orientações para a melhor forma de realizar as atividades laborais e a própria modificação do trabalho. Nesse sentindo, é necessária a atuação de diversos profissionais, como médicos, engenheiros, psicólogos, terapeutas ocupacionais, fisioterapeutas, ergonomistas, assistentes sociais, dentre outros, para garantir a análise global da problemática (SATO, 2001; SETTIMI et al., 1998).

As atividades em grupos complementando os procedimentos terapêuticos com pacientes com LER/ DORT têm sido utilizadas desde o início dos anos 1990 (LIMA; OLIVEIRA, 1995). Elas têm possibilitado que os trabalhadores acometidos saibam lidar de forma mais autônoma com o seu quadro clínico e com as limitações associadas, que eles modifiquem sua forma de trabalhar e de realizar as atividades de vida diária e que também possam amenizar o sofrimento advindo da doença e da culpabilização a eles atribuída pelo adoecimento do qual são vítimas.

As vivências grupais permitem ao trabalhador transformar percepções individuais em percepções coletivas, a partir da identificação de seus próprios processos com os dos outros participantes. Dessa forma, favorecem o estabelecimento de relações do seu próprio adoecimento com o processo de trabalho e facilitam a compreensão de que o processo de adoecimento é mais do que um processo individual e, sim, decorrente do próprio trabalho (SATO et al., 1993).
Apesar dos programas de tratamentos e reabilitação de pacientes com LER/DORT indicarem uma abordagem multidisciplinar, a fisioterapia é muitas vezes o primeiro e único procedimento terapêutico acessível e pode ser uma etapa de longa duração no tratamento desses trabalhadores (ASSUNÇÃO, 2001).

A utilização de recursos físicos é importante para o controle da dor dos pacientes com LER/DORT. Os recursos analgésicos devem ser associados à cinesioterapia para proporcionar a redução do edema e da inflamação, a melhora das condições circulatórias, o relaxamento da musculatura, a amenização da dor e uma potencialização da capacidade funcional destes pacientes (YENG, 1995).

Acredita-se que, no tratamento fisioterapêutico, a abordagem em grupo também poderia potencializar os efeitos dos recursos físicos utilizados, acentuando a melhora do quadro clínico dos pacientes (SIQUEIRA; QUEIROZ, 2001).

A cinesioterapia em grupo faz com que o paciente aprenda a assumir parte da responsabilidade de seu próprio exercício, adquira confiança no tratamento, compreenda a dimensão coletiva do seu adoecimento, rompa com o isolamento, muitas vezes provocado pela doença, e perceba que, ao mesmo tempo em que precisa de ajuda, pode auxiliar outros membros do grupo (GARDINER, 1993; ZIMERMAN, 1997). Dessa forma, num tratamento fisioterapêutico dos trabalhadores com LER/DORT, se restabeleceria o caráter social do processo de adoecimento, reduzindo o foco no sintoma, tal como é feito nos tratamentos tradicionais.

A participação nos grupos oferece, ainda, maior reconhecimento social, que pode auxiliar o portador de LER/DORT a ficar mais confiante em si, a conviver melhor com sua situação clínica durante o tratamento, com suas limitações, a se preparar melhor para o retorno ao trabalho ou a procurar novas formas de inserção profissional (LIMA; OLIVEIRA, 1995).

O objetivo deste artigo é avaliar os benefícios da associação do tratamento cinesioterapêutico com as dinâmicas de grupo em uma abordagem mais integral do processo saúde-doença no processo de recuperação e reabilitação de pacientes com LER/DORT, em relação à funcionalidade e ao processo de retorno ao trabalho.

\section{Método}

Trata-se de um estudo clínico comparando dois tipos de tratamento: a intervenção cinesioterapêutica individual com a intervenção cinesioterapêutica em grupo.

\section{Local e sujeitos}

Este estudo foi desenvolvido em 2008, em dois Centros de Referência em Saúde do Trabalhador, um localizado no município de Santo André e o outro, no município de São Paulo, no Estado de São Paulo, Brasil. 
Os critérios de inclusão dos sujeitos nesta pesquisa foram: trabalhadores (homens e mulheres) que possuíam uma ou múltiplas patologias em membros superiores, com nexo com trabalho estabelecido (diagnóstico clínico de LER/DORT) e com a Comunicação do Acidente de Trabalho emitida, independentemente da situação ocupacional em que se encontravam (empregados, afastados ou desempregados).

Foram selecionados 44 pacientes com diagnóstico clínico de LER/DORT em membros superiores, que aguardavam atendimento fisioterapêutico nestes serviços, caracterizando uma amostra de conveniência. Destes, 24 pacientes concordaram em participar do estudo e foram aleatoriamente distribuídos para os dois tipos de intervenção: tratamento cinesioterapêutico em grupo $(n=12)$ e tratamento cinesioterapêutico individual $(n=12)$.

Todos os pacientes assinaram um termo de consentimento livre e esclarecido, conforme resolução 196/96 do Conselho Nacional de Saúde. O estudo foi aprovado pelo Comitê de Ética em Pesquisa do Hospital das Clínicas, da Faculdade de Medicina da Universidade de São Paulo, e pelo Comitê de Ética em Pesquisa da Secretaria Municipal de Saúde da Cidade de São Paulo.

\section{Procedimentos}

Os procedimentos realizados nesta pesquisa foram divididos em 3 etapas: avaliação inicial, intervenção individual ou em grupo e uma avaliação final. Estes procedimentos estão explicados nos tópicos a seguir.

\section{Avaliação:}

Os pacientes foram avaliados individualmente em uma sessão antes e em outra após o período de tratamento, com os mesmos instrumentos de avaliação.

Um protocolo de avaliação fisioterapêutica em saúde do trabalhador que investiga a história clínica, ocupacional e o comprometimento físico foi utilizado para o acompanhamento desses indivíduos durante todo procedimento de pesquisa e para a caracterização da amostra.

A versão brasileira do questionário DASH (Disabilities of the Arm, Shoulder and Hand) foi utilizada para mensurar o impacto das intervenções no estado funcional dos membros superiores dos pacientes com LER/DORT (ORFALE et al., 2005; CAMARGO et al., 2007). Este questionário contém 30 questões que exploram a funcionalidade e os sintomas em membros superiores; cada questão tem cinco possibilidades de resposta, variando entre não haver dificuldade e não conseguir realizar a atividade questionada, havendo uma pontuação de 1 a 5 . A pontuação final do questionário varia de 0 a 100, sendo obtida pela aplicação de uma fórmula, sendo que o valor 100 indica uma incapacidade física dos membros superiores para as atividades de vida diária e a de trabalho. O módulo ocupacional deste questionário também foi utilizado para verificar a capacidade funcional para a atividade de trabalho.
Uma entrevista semiestruturada com questões predeterminadas foi realizada com os pacientes, apenas após o tratamento, com a finalidade de analisar, sob o ponto de vista qualitativo, os efeitos das intervenções em relação à melhora do quadro clínico, à percepção sobre seu adoecimento, sobre as modificações da intervenção em suas vidas e as perspectivas para o retorno ao trabalho.

\section{Intervenção:}

O tratamento cinesioterapêutico em grupo foi denominado de intervenção grupal e o tratamento cinesioterapêutico realizado com uma única pessoa, de intervenção individual. Os dois tipos de intervenção ocorreram em 10 sessões, com uma frequência de 2 vezes por semana.

O protocolo de cinesioterapia foi o mesmo para as duas intervenções: individual e grupal. Os exercícios foram baseados em técnicas de autoalongamento, fortalecimento muscular, mobilização articular ativa, facilitação neuromuscular proprioceptiva, reeducação postural e exercícios respiratórios (KISNER; COLBY, 1998; BANDY; SANDERS, 2003). Assim, foi estabelecida uma sequência de exercícios para cada sessão, que foi aplicada da mesma forma e com as mesmas orientações nos dois tipos de intervenções (Quadro 1).

Os exercícios de autoalongamento eram mantidos por 20 segundos com séries de 5 repetições, e os exercícios de fortalecimento eram realizados com séries de 10 repetições sem carga. O protocolo estabeleceu a introdução progressiva dos exercícios para respeitar a evolução clínica dos pacientes.

Após os exercícios, eram abordados temas considerados importantes no tratamento dos pacientes com LER/DORT, como as causas do adoecimento, a influência da doença nas limitações impostas pelo quadro clínico, as modificações e as novas alternativas para realizar as atividades de vida diária necessárias para o controle do quadro e a garantia da funcionalidade, as dificuldades do tratamento e do controle do quadro doloroso, o impacto do adoecimento na vida familiar, a participação do paciente no tratamento e o retorno ao trabalho (Quadro 1).

A fim de padronizar essa abordagem nas duas intervenções, foi criada uma estratégia para possibilitar o processo de reflexão destes aspectos nas intervenções individuais e em grupo com a leitura de historietas clínicas.

A palavra historieta refere-se à narração de uma história pequena, curta. Foi estabelecido um tema para cada sessão e para cada tema encontrado uma narração feita por um paciente com LER/DORT (historieta clínica) que trouxesse para a discussão o tema equivalente. Essas narrações foram retiradas de livros, e as entrevistas publicadas em jornais e revistas.

No final de cada sessão, essa historieta clínica era lida para o paciente que participava da intervenção individual para que o mesmo refletisse sobre o assunto e, se fosse seu desejo, poderia comentar a leitura com a fisioterapeuta. 
Quadro 1 Descrição resumida dos exercícios utilizados no protocolo de cinesioterapia e dos temas das historietas clínicas

\begin{tabular}{|c|c|}
\hline Resumo do protocolo de cinesiosterapia & $\begin{array}{l}\text { Temas } \\
\text { (historieta clínica) }\end{array}$ \\
\hline - Exercícios respiratórios. & - O quadro doloroso e suas exacerbações. \\
\hline $\begin{array}{l}\text { - Autoalongamento dos músculos: serrátil anterior, eleva- } \\
\text { dor da escápula, peitoral maior, flexores e extensores } \\
\text { de punho, intrínsecos dos dedos das mãos, inclinadores } \\
\text { laterais, rotadores e flexores de coluna cervical. }\end{array}$ & $\begin{array}{l}\text { - O afastamento do ambiente de trabalho. } \\
\text { - As atividades da vida diária. } \\
\text { - As atividades de lazer. }\end{array}$ \\
\hline $\begin{array}{l}\text { - Mobilização ativa de coluna cervical, ombros, punhos, } \\
\text { coluna lombar e pelve. }\end{array}$ & $\begin{array}{l}\text { - As relações familiares e com os amigos. } \\
\text { - O tratamento e as diversas técnicas terapêuticas. }\end{array}$ \\
\hline $\begin{array}{l}\text { - Fortalecimento dos músculos rombóides, musculatura } \\
\text { flexora, abdutora e rotadora de ombros e da musculatura } \\
\text { intrínseca das mãos. }\end{array}$ & $\begin{array}{l}\text { - O papel da previdência social na recuperação de pa- } \\
\text { cientes com LER/DORT. }\end{array}$ \\
\hline $\begin{array}{l}\text { - Autopostura: cadeia posterior, cadeia anterior de membro } \\
\text { superior e cadeia anterointerna de quadril. }\end{array}$ & $\begin{array}{l}\text { - O retorno ao trabalho. } \\
\text { - O papel do paciente na recuperação do trabalho doente. }\end{array}$ \\
\hline $\begin{array}{l}\text { - Facilitação neuromuscular proprioceptiva: diagonais de } \\
\text { membros superiores. }\end{array}$ & $\begin{array}{l}\text { - A fisioterapia e o fisioterapeuta na recuperação na } \\
\text { LER/DORT. }\end{array}$ \\
\hline
\end{tabular}

Para os pacientes que participaram da intervenção em grupo, a mesma estratégia de leitura das historietas acontecia, entretanto, os mesmos, em um processo interativo, eram convidados a comentar e a discutir entre eles o tema apresentado.

Em ambas as intervenções, a fisioterapeuta coordenou as discussões dos temas, não interferindo no desenrolar das mesmas, respondendo apenas algumas dúvidas de ordem fisiopatológica relacionadas a aspectos técnicos da doença, mantendo-se como mediadora das discussão ou dos comentários propostos pelas historietas.

\section{Análise dos dados}

As variáveis idade e tempo de início dos sintomas foram analisadas pelo teste t de Student. O número de indivíduos para as categorias sexo, escolaridade e situação ocupacional foi comparado utilizando-se o teste de Fischer. Já para as categorias atividade de trabalho e diagnóstico clínico foi utilizada a descrição simples do número de indivíduos por subcategoria.

O teste de Wilcoxon foi utilizado para analisar a pontuação do questionário DASH e do módulo ocupacional do questionário DASH, antes e após as intervenções.

O nível de significância adotado foi de p<0,05.

As entrevistas foram gravadas e transcritas na íntegra e foram analisadas seguindo o modelo de análise de conteúdo proposto por Minayo (1992). Nesta forma de análise, através de uma leitura flutuante, buscou-se detectar, em um primeiro momento, temas que se repetiam nas entrevistas para que depois estes fossem agrupados e transformados em categorias que pudessem ser contrapostas, comparadas e analisadas.

\section{Resultados}

\section{Perfil da população estudada}

As variáveis idade, sexo, escolaridade, situação ocupacional e tempo de início dos sintomas apresentaram $\mathrm{p}>0,05$, não havendo diferenças entre os participantes das intervenções individual e em grupo (Tabela 1).

Na Tabela 2, estão descritas as atividades de trabalho dos pacientes por intervenção e pode-se identificar uma diversidade de ocupações em ambas as intervenções. Ainda na Tabela 2, é possível verificar os diagnósticos clínicos dos pacientes, nos quais as patologias de ombro foram encontradas com maior prevalência entre os participantes das duas intervenções.

\section{Avaliação da funcionalidade/questionário DASH}

A Tabela 3 mostra a mediana de pontuação do questionário DASH e do módulo ocupacional do questionário DASH dos pacientes das intervenções individual e em grupo, antes e depois do tratamento.

De acordo com essa tabela, pode ser observado que houve uma redução da pontuação do questionário DASH em ambas as intervenções, entretanto essa diminuição não foi considerada significante.

A avaliação da funcionalidade em relação à atividade de trabalho, através do módulo ocupacional do questionário DASH, também revelou que não houve diferenças significantes na mediana da pontuação antes e após as intervenções nesta amostra (Tabela 3). 
Tabela 1 Caracterização da amostra dos pacientes com LER/DORT, submetidos à intervenção individual e em grupo, segundo idade, sexo, escolaridade, situação ocupacional e tempo de início dos sintomas

\begin{tabular}{|c|c|c|}
\hline Variáveis & Intervenção individual & Intervenção em grupo \\
\hline \multicolumn{3}{|l|}{ Idade $(p=0,38)$} \\
\hline Média (em anos) & $48,67 \pm 7,35$ & $45,75 \pm 8,12$ \\
\hline \multicolumn{3}{|l|}{$\operatorname{Sexo}(p=0,31)$} \\
\hline Feminino & 11 & 08 \\
\hline Masculino & 01 & 04 \\
\hline \multicolumn{3}{|l|}{ Escolaridade $(p=0,66)$} \\
\hline Ensino fundamental & 7 & 9 \\
\hline Ensinos médio e superior & 5 & 3 \\
\hline \multicolumn{3}{|l|}{ Situação ocupacional $(p=0,37)$} \\
\hline Em atividade ou com afastamento & 7 & 10 \\
\hline Desempregado & 5 & 2 \\
\hline \multicolumn{3}{|l|}{ Tempo de início dos sintomas $(\mathrm{p}=0,28)$} \\
\hline Média (em anos) & $4,5 \pm 2,64$ & $5,5 \pm 1,73$ \\
\hline
\end{tabular}

Os valores estão apresentados em número de indivíduos ou valor médio com respectivo desvio padrão.

Tabela 2 Distribuição do número de pacientes com LER/DORT, submetidos à intervenção individual e em grupo, por atividade de trabalho e por diagnóstico clínico

\begin{tabular}{lcc}
\hline & Intervenção individual & Intervenção em grupo \\
\hline Atividade de trabalho & 01 & 0 \\
Bancário & 03 & 03 \\
Costureira & 01 & 0 \\
Cozinheira & 04 & 03 \\
Limpeza/camareira/passadeira & 0 & 05 \\
Metalúrgicos/indústria & 03 & 01 \\
Outros & & \\
Diagnóstico Clínico* & 03 & 02 \\
Síndrome do Túnel do Carpo & 02 & 01 \\
Cervicobraquialgia & 02 & 04 \\
Tenossinovites de punhos & 02 & 01 \\
Epicondilites & 09 & 08 \\
Síndrome do impacto/ Síndrome do manguito & & 06 \\
rotador/Tendinite do m. Supraespinhoso & 07 & \\
Bursites de ombro & & 03 \\
\hline
\end{tabular}

*Os pacientes podem apresentar mais de um diagnóstico clínico. 
Tabela 3 Mediana da pontuação do questionário DASH e do módulo ocupacional do questionário DASH, dos pacientes com LER/DORT, antes e depois das 10 sessões de intervenção individual e em grupo, São Paulo, 2008

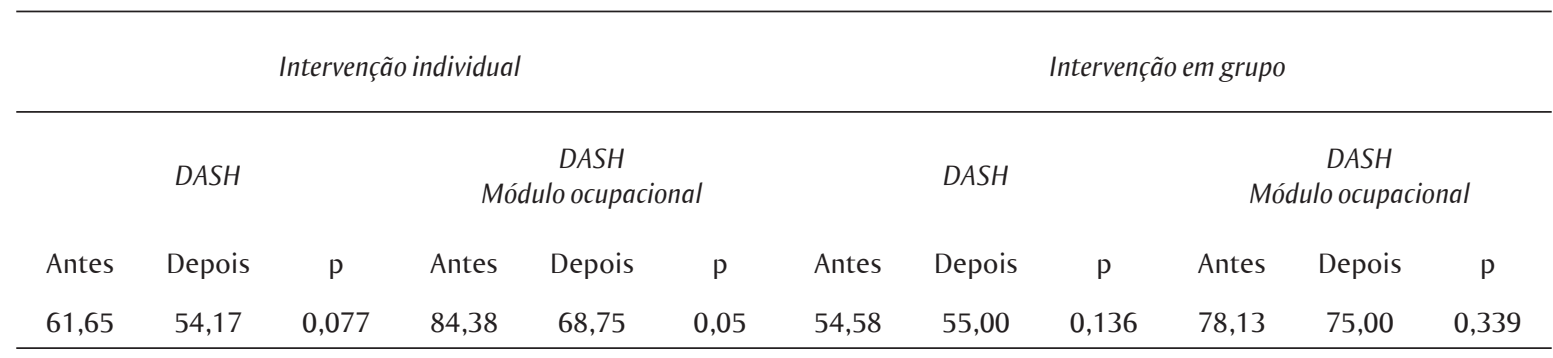

Nível de significância $\mathrm{p}<0,05$

\section{Análise das entrevistas}

A análise das entrevistas permitiu identificar sete temas relacionados aos dois tipos de intervenções realizadas pelos pacientes: modificações no quadro clínico, modificações na vida cotidiana, tratamento e intervenção em grupo, tratamento e historietas clínicas, tratamento cinesioterapêutico, relação terapeuta-paciente e perspectivas para o retorno ao trabalho, como pode ser visto no Quadro 2.

A análise destes temas, oriundos dos depoimentos dos participantes das duas intervenções, revelou melhora do quadro clínico e de maneira semelhante entre eles, como ilustram os trechos abaixo que identificam no final o tipo de intervenção (Individual - I ou Grupal - G) e classificam os pacientes entrevistados através de um numero (p.): "Agora eu tenho menos dor, não fico reclamando tanto" (I, p. 6). "Eu não sinto dor com frequência como no caso quando eu cheguei” (G, p. 3).

A análise das entrevistas permitiu identificar que os dois tipos de intervenção produziram ganhos indiretos, na vida cotidiana desses pacientes, em três aspectos: no ritmo de execução das atividades de vida diária, nas atividades de lazer e nas relações interpessoais.

As entrevistas apontam que, após a intervenção individual e em grupo, ocorreu uma diminuição no ritmo de execução das atividades de vida diária, ou seja, os participantes estão planejando as atividades e executando-as de forma mais lenta.

Associado a isso, identificou-se que os pacientes estavam desenvolvendo uma nova noção de seus limites e passavam a respeitar os seus novos limites: "Procuro fazer aos poucos. Hoje faço uma coisa, amanhã, outra” (I, p. 9). "Para mim tá mais fácil assim, porque eu procuro manter a casa todo dia organizada” (G, p. 9).

Segundo o relato dos pacientes, as intervenções produziram efeitos diferentes na melhora da funcionalidade. No discurso de alguns pacientes participantes da intervenção individual, estava presente a dificuldade para a realização de algumas atividades:
"Eu fico mais com o braço imobilizado, eu movimento pouco. Faço alguma coisa básica, mas muita coisa não dá pra fazer” (I, p. 8).

Já na fala dos participantes que participaram da intervenção em grupo, houve uma percepção de melhora funcional: "Consigo fazer com mais facilidade, não com tanta agilidade” (G, p. 10).

Embora as historietas clínicas não tenham sido valorizadas em si pelos pacientes submetidos à intervenção em grupo, a possibilidade de discussão dos assuntos despertados a partir das leituras permitiu a troca de experiências vivenciadas entre os integrantes do grupo. Este nos parece ser o aspecto mais relevante da abordagem cinesioterapêutica em grupo: "Não só o tratamento, mas a leitura que você fazia me ajudou bastante [...]. As histórias de outros pacientes que já tinham passado uma experiência de vida...” (G, p. 9).

Já no tratamento individual, as historietas representaram uma possibilidade de compartilhar as experiências de outros pacientes: "[...] Eu já senti muitas coisas dessa daí que você leu” (I, p. 10).

As dinâmicas do grupo possibilitaram, entre os seus integrantes, uma relação interpessoal, que os estimulava na execução dos exercícios e que facilitou uma tomada de consciência sobre o adoecimento e o processo de recuperação.

Os pacientes submetidos à intervenção grupal apresentaram um maior vínculo terapeuta-paciente, que parece ter ocorrido através da maior compreensão e do domínio do fisioterapeuta sobre os processos de recuperação e reabilitação desses indivíduos. Estes pacientes descreveram ter mais autonomia sobre seus tratamentos e, ao mesmo tempo, uma relação de proximidade com o fisioterapeuta.

No tratamento individual, a relação terapeuta-paciente se estabeleceu de forma mais dependente. A relação de confiança e compromisso também ocorreu nessa relação, mas os pacientes associaram uma maior transferência da melhora clínica ao profissional. 
Quadro 2 Resumo das informações obtidas pelas entrevistas dos pacientes com LER/DORT, na intervenção individual e em grupo, decorrentes da análise de conteúdo em cada tema

\begin{tabular}{|c|c|c|}
\hline Temas & Intervenção Individual & Intervenção em Grupo \\
\hline Modificações no quadro clínico & $\begin{array}{l}\text { - Melhora da dor } \\
\text { - Mais preparação para lidar com os } \\
\text { sintomas }\end{array}$ & $\begin{array}{l}\text { - Diminuição da frequência dos sinto- } \\
\text { mas álgicos } \\
\text { - Melhora da funcionalidade }\end{array}$ \\
\hline Modificações na vida cotidiana & $\begin{array}{l}\text { - Diminuição do ritmo de execução das } \\
\text { AVDs** } \\
\text { - Dificuldade para realizar atividades } \\
\text { - Melhora nas relações familiares }\end{array}$ & $\begin{array}{l}\text { - Diminuição do ritmo de execução das } \\
\text { AVDs* } \\
\text { - Percepção de melhora funcional } \\
\text { - Melhora nas relações familiares e } \\
\text { ampliação da rede social }\end{array}$ \\
\hline O tratamento e a intervenção em grupo & & $\begin{array}{l}\text { - Consciência sobre o processo de } \\
\text { recuperação } \\
\text { - Percepção de outros grupos sociais }\end{array}$ \\
\hline O tratamento e as historietas clínicas & $\begin{array}{l}\text { - Possibilidade de reflexões sobre o } \\
\text { adoecimento }\end{array}$ & $\begin{array}{l}\text { - Possibilidade de discussão a partir das } \\
\text { leituras }\end{array}$ \\
\hline O tratamento cinesioterapêutico & $\begin{array}{l}\text { - Exercício para o controle do quadro } \\
\text { doloroso }\end{array}$ & $\begin{array}{l}\text { - Exercício para o controle do quadro } \\
\text { doloroso } \\
\text { - Associação do exercício com a } \\
\text { melhora }\end{array}$ \\
\hline $\begin{array}{l}\text { A relação terapeuta-paciente nas } \\
\text { intervenções }\end{array}$ & $\begin{array}{l}\text { - Relação de confiança e compromisso } \\
\text { - Dependência no tratamento } \\
\text { - Relação humanizada }\end{array}$ & $\begin{array}{l}\text { - Relação de confiança e compromisso } \\
\text { - Autonomia no tratamento } \\
\text { - Relação humanizada }\end{array}$ \\
\hline Perspectivas para o retorno ao trabalho & $\begin{array}{l}\text { - Desejo de retorno ao trabalho } \\
\text { - Retorno vinculado à recuperação física } \\
\text { - Não melhora da capacidade laboral }\end{array}$ & $\begin{array}{l}\text { - Desejo de retorno ao trabalho } \\
\text { - Retorno vinculado a modificações no } \\
\text { ambiente de trabalho } \\
\text { - Não melhora da capacidade laboral }\end{array}$ \\
\hline
\end{tabular}

*AVDs: Atividades da vida diária

Os discursos dos pacientes obtidos a partir das entrevistas apontaram que tanto o tratamento cinesioterapêutico individual, quanto aquele associado às dinâmicas de grupo não foram suficientes para melhorar a capacidade de retorno ao trabalho, mas o desejo e a mobilização para o retorno ao trabalho esteve presente nos discursos dos participantes em ambas intervenções.

Um fator limitante para o retorno ao trabalho identificado nas entrevistas, além dos aspectos sociais decorrentes do adoecimento, foi a presença ou a recordação da dor e também o fato de que voltar ao trabalho significava reviver toda a situação que ocasionou o adoecimento: “[...] Quando eu lembro do trabalho... acabo me lembrando da dor" (I, p. 7). "Não tinha esperança em voltar a trabalhar, só pelo fato de todo dia conviver com a dor" (G, p. 9).

Os pacientes que passaram pelo processo grupal relataram que o retorno ao trabalho somente seria possível se houvesse modificações nas condições de trabalho, principalmente a diminuição do ritmo e a introdução de pausas na atividade de trabalho: "Na mesma, posso até continuar. Assim, tendo pausas e não sendo mais tão corrido. Em outro ritmo" (G, p. 9).

Já entre os pacientes da intervenção individual ocorreu uma associação do retorno ao trabalho somente à recuperação física: "Eu espero melhorar e arrumar um serviço. Poder ficar boa de tudo. Mas, trabalhar sem sentir dor” (I, p. 6). "[...] Eu creio que na minha função mesmo eu não vou conseguir” (I, p. 1).

\section{Discussão}

Os estudos clínicos envolvendo pacientes com LER/ DORT comumente esbarram na dificuldade do número de participantes e da constituição de amostras homogêneas.

No presente estudo, mesmo se utilizando de uma amostra de conveniência na tentativa de conseguir um número maior de participantes, foi observada uma pequena adesão à pesquisa. Este fato pode estar associado ao receio ou ao medo de participar de procedimentos 
talvez ainda desconhecidos por esta população. Outra explicação é que não é raro os pacientes com LER/DORT apresentarem restrições financeiras para o transporte urbano, dificultando a participação nos tratamentos.

A dificuldade em constituir amostras homogêneas em pesquisas com LER/DORT pode estar associada à quantidade ampla de atividades de trabalho que apresentam os vários fatores causais para estes acometimentos, às múltiplas queixas dos pacientes, aos diversos diagnósticos clínicos e às diversas regiões acometidas.

Apesar disso, os participantes desta pesquisa não apresentavam diferenças significantes em sua caracterização, mas é importante ressaltar que, devido ao número pequeno de participantes deste estudo, os resultados gerados são específicos para esta amostra.

Neste estudo, foi observado que as intervenções (individual e grupal) não produziram efeitos diferentes e significativos, no estado funcional dos membros superiores, para as atividades de vida diária, segundo mensuração realizada através do questionário DASH, embora a percepção dos pacientes tenha sido contrária, identificando melhora da funcionalidade e ganhos indiretos nas atividades cotidianas, nas duas intervenções.

Pransky et al. (1997) descreveram que, muitas vezes, a mensuração clínica da funcionalidade através de instrumentos não condiz com a avaliação das atividades realizadas em casa ou no trabalho pelos pacientes.

O questionário DASH é um instrumento indicado para a percepção de mudanças na funcionalidade de membros superiores, mas talvez o período curto de tratamento tenha sido insuficiente para trazer benefícios quantificáveis por este instrumento.

Outro aspecto relevante é que o questionário DASH aborda o grau de dificuldade para a execução das atividades. Por se tratar de pacientes crônicos, a dificuldade pode não ter se modificado, mas as estratégias para realização das atividades sim. Essas estratégias não foram mensuradas no questionário, apenas nas entrevistas.

Essa constatação reforça a ideia apontada por Sluiter e Frings-Dresen (2008) de que os efeitos quantitativos de uma intervenção clínica podem não revelar o conjunto do impacto do tratamento nos pacientes com LER/DORT. É preciso conhecer os aspectos subjetivos e a percepção dos indivíduos do seu processo de recuperação e reabilitação para melhor avaliar os vários aspectos da eficácia dos procedimentos clínicos.

Em um estudo que comparou os efeitos da fisioterapia individual e dos exercícios em grupo em trabalhadores com mialgia em ombros e cervical, foi verificado que, nos dois tipos de intervenção, houve uma melhora do quadro clínico. Porém, os sujeitos que receberam o tratamento em grupo estavam mais satisfeitos com seus, estados, de saúde e também mantiveram a melhora por mais tempo do que os pacientes da intervenção individual (VASSELJEN JR.; JOHANSEN; WESTGAARD, 1995).
A análise qualitativa possibilitou identificar que as reflexões e as trocas de experiências sobre as implicações da dor crônica em diversos aspectos da vida dos pacientes com LER/DORT, ocorridas no processo grupal, trouxeram uma nova compreensão sobre o estado de saúde desses indivíduos. Essa maior compreensão permitiu uma reorganização de suas emoções e do convívio com o sofrimento e possibilitou a identificação de uma resposta mais favorável ao tratamento, mesmo não havendo uma resposta clínica de melhora funcional relevante.

Os pacientes que passaram pela intervenção em grupo parecem estar mais motivados na busca da autonomia e mais estáveis emocionalmente do que os pacientes que se submeteram à intervenção individual.

Essa diferença pode ser decorrente da ausência de trocas, que ocorreu na intervenção individual. Os participantes que passaram por esta intervenção refletiram sobre todos os temas referentes ao adoecimento, mas não escutaram as diversidades dos quadros clínicos, das condições de trabalho e das dificuldades vivenciadas pelos participantes do processo grupal.

Siqueira e Queiroz (2001) relataram a experiência dos profissionais de um serviço de saúde do trabalhador do Município de São Paulo, na qual identificaram que os procedimentos terapêuticos individuais e isolados de fisioterapia, terapia ocupacional e psicologia não respaldavam todas as necessidades dos trabalhadores com LER/DORT. Dessa forma, reorganizaram o atendimento dessas pessoas através de uma abordagem mais integrada, visando aumentar as trocas de experiência que facilitassem tanto a recuperação e sua inclusão em um novo trabalho, quanto o retorno à mesma função.

Essas autoras fizeram uso de dinâmicas grupais para favorecer uma intervenção mais integrada e obtiveram como resultados a diminuição da sintomatologia e das questões emocionais, a sensação de prazer durante os encontros, a recontextualização da capacidade laboral e um novo posicionamento como cidadãs dessas pessoas que passaram a entender que o problema vivenciado não era individual, e sim coletivo. Assim, compreende-se que essa abordagem possibilitou, além da recuperação física do trabalhador, maior compreensão social do problema, que extrapolou os efeitos da clínica tradicional e se estendeu a outros aspectos da vida dessas pessoas (SIQUEIRA; QUEIROZ, 2001).

O processo de retorno ao trabalho e de permanência para os pacientes com LER/DORT é bastante complexo e um dos mais importantes aspectos da prevenção, do tratamento e da reabilitação de trabalhadores portadores desse tipo de lesão (GRAVINA; ROCHA, 2006; NIEUWENHUIJSEN et al., 2003; LANCMAN, 2001). Os resultados das duas intervenções em relação a essa questão foram muito superficiais. Enquanto a avaliação quantitativa demonstrou que as intervenções não produziram efeitos no aumento da funcionalidade para a atividade de trabalho, a avaliação qualitativa apontou que as dificuldades encontradas pelos pacientes para que esse retorno aconteça vão além dos sintomas físicos ou da funcionalidade. 
Feuerstein et al. (1993) demonstraram que um programa de reabilitação multidisciplinar de pacientes crônicos com LER/DORT auxilia no retorno ao trabalho, mas revelou que são necessárias também intervenções nas condições de trabalho e o acompanhamento dos trabalhadores nesse retorno para garantir uma volta efetiva e permanente.

Gravina e Rocha (2006) publicaram um estudo sobre o processo de retorno ao trabalho dos bancários e observaram que, para readaptar o paciente com LER/ DORT ao trabalho, é preciso um envolvimento efetivo das empresas e dos trabalhadores.

Para Yeng et al. (2001), o retorno ao trabalho é dificultado pela desatualização das habilidades e dos conhecimentos adquiridos, antes do afastamento, pelos déficits funcionais e pelo medo real e/ou infundado de novos episódios de lesão.

Estas informações foram sustentadas neste estudo, pois as limitações funcionais, especialmente para a atividade de trabalho, o medo de novos episódios de adoecimento e a incerteza de como seriam recebidos em seus locais de trabalho, no caso de alta, estiveram presentes nos discursos dos pacientes, após o tratamento, nas duas formas de intervenção.

As autoras Gravina e Rocha (2006) discutem que a presença de dor e a dificuldade de consciência das limitações funcionais dificultam o retorno ao trabalho. Opsteegh et al. (2009), que investigaram os determinantes do retorno ao trabalho em pacientes com lesões em mãos, também concluíram que a dor foi um fator dificultador do retorno ao trabalho.

No estudo de Takahashi e Canesqui (2003) sobre a avaliação da efetividade de um serviço de saúde na reabilitação de pacientes com LER/DORT, as autoras consideraram imprescindível para o retorno ao trabalho um atendimento terapêutico que resgate a autonomia funcional e o equilíbrio emocional.

Um fato é que as falas dos pacientes com LER/DORT que participaram dessa pesquisa ressaltaram as reflexões sobre o trabalho enquanto centralizador social, que mantém o indivíduo no mundo, e como fonte de identidade psicossocial (NUNES; ANDRADE, 2007). Esses elementos demonstram que o trabalho em si e a sua importância global na vida das pessoas merece mais destaque, e um tempo maior de abordagem dentro das sessões, independentemente do tipo de intervenção (individual ou em grupo).

Outro aspecto relevante e que pode ter limitado os efeitos das intervenções é o número de sessões previstas neste protocolo de tratamento.

\section{Referências}

ASSUNÇÃO, A. A. Os DORT e a dor dos DORT. In: CONGRESSO DA ASSOCIAÇÃO NACIONAL DE MEDICINA DO TRABALHO, 11., 2001, Belo Horizonte. Anais... Belo Horizonte: ANAMT, 2001. 1 CD-ROM.
A escolha do número de sessões foi baseada na prática clínica realizada na maioria das clínicas de fisioterapia, que, por motivos de organização financeira com os planos de saúde, predefinem os tratamentos em 10 sessões. Os serviços públicos de fisioterapia em saúde do trabalhador, por sua vez, para solucionar a grande demanda de pacientes e permitir maior rotatividade, estipulam prazos e números de sessões definidas para a finalização do tratamento.

Nos estudos citados por Konijnemberg et al. (2001), em uma revisão sistemática de tratamento conservador para LER/DORT, não havia um consenso sobre o estabelecimento do número de sessões para garantir a eficácia do tratamento e a recuperação dos pacientes.

No presente estudo, a necessidade de continuidade do tratamento foi percebida pelos pacientes que se submeteram aos dois tipos de intervenções, mostrando que a quantidade de sessões deve ser maior do que a estipulada nesta pesquisa, tanto nas intervenções individuais, quanto nas grupais.

Assim, o sucesso ou não do processo de reabilitação e da reinserção do trabalhador em seu posto de trabalho, ou em outro, após a recuperação ou o controle do quadro clínico, tem sido um enigma, havendo a necessidade de outros estudos que auxiliem no entendimento e no fortalecimento do processo de retorno ao trabalho de pacientes com LER/DORT.

\section{Conclusão}

O número pequeno de participantes e o curto tempo de acompanhamento dos tratamentos podem ter limitado os resultados sobre a funcionalidade e o retorno ao trabalho dos pacientes do estudo. Mas foi possível perceber que o tratamento cinesioterapêutico individual e o associado às dinâmicas de grupo permitiu uma abordagem mais global do processo de adoecimento, recuperação e reabilitação do paciente com LER/DORT, mesmo não sendo suficiente para garantir o retorno ao trabalho.

Este estudo trouxe uma importante fundamentação para as ações dos fisioterapeutas que querem atuar em grupo, especialmente na área de saúde do trabalhador. Revelou que a possibilidade de reflexão criada nas duas intervenções, individual e grupal, foi indispensável para as modificações na percepção do quadro clínico e da funcionalidade dos pacientes com LER/DORT e que as estratégias de utilização das historietas clínicas podem ser incorporadas na prática clínica dos fisioterapeutas durante o atendimento desses pacientes como forma de enriquecer os tratamentos e de aumentar a relação terapeuta-paciente.

BANDY, W. D.; SANDERS, B. Exercícios terapêuticos: técnicas para intervenção. Rio de Janeiro: Guanabara Koogan, 2003.

BRASIL. Ministério da Saúde. Organização PanAmericana da Saúde. Doenças relacionadas ao 
trabalho: manual de procedimentos para os serviços de saúde. Brasília: Minsitério da Saúde, 2001. (Série A. Normas e manuais técnicos, n. 114).

CAMARGO, P. R. et al. Pain in workers with shoulder impingement syndrome: an assessment using the DASH and McGill pain questionnaires. Revista Brasileira de Fisioterapia, São Carlos, v. 11, n. 2, p. 161-67, mar./abr. 2007.

FEUERSTEIN, M. et al. Multidisciplinary rehabilitation of chronic work-related upper extremity disorders. Journal Occupational Medicine, United States, v. 35, n. 4, p. 396-403, 1993.

GARDINER, D. Manual de terapia por exercícios. São Paulo: Livraria Editora Santos, 1993.

GRAVINA, M. E. R.; ROCHA, L. E. Lesões por esforços repetitivos em bancários: reflexões sobre o retorno ao trabalho. Cadernos de Psicologia Social e do Trabalho, São Paulo, v. 9, n. 2, p. 41-55, dez. 2006.

KISNER, C.; COLBY, L. A. Exercícios terapêuticos: fundamentos e técnicas. 3. ed. São Paulo: Manole, 1998.

KONIJNENBERG, H. S. et al. Conservative treatment for repetitive strain injury. Scandinavian Journal Work Environ \& Health, Finland, v. 27, p. 299-310, Oct. 2001.

LANCMAN, S. Les Lesion par efforts repetitifs au Bresil: l'expression de la soufrance dans le travail et le peur du choage. In: COLLOQUE INTERNATIONAL DE PSYCHODYNAMIQUE ET PSYCHOPATHOLOGIE DU TRAVAIL LA PEUR ET L'ACTION DANS LE CHAMP DU TRAVAIL, 3., 2001, Paris. Anais... Paris: CNAM, 2001. v. 1, p. 107-113.

LIMA, A. B.; OLIVEIRA, F. Abordagem psicossocial da LER: ideologia da culpabilização e grupos de qualidade de vida. In: CODO, W. E.; ALMEIDA, M. C. C. (Org.) Lesões por Esforços Repetitivos L. E. R. Diagnóstico, tratamento e prevenção - uma abordagem interdisciplinar. Petrópolis: Vozes, 1995. p. 159-163.

MINAYO, M. C. S. O desafio do conhecimento: pesquisa qualitativa em saúde. São Paulo. Rio de Janeiro: Hucitec-Abrasco, 1992.

NIEUWENHUIJSEN, K. et al. Quality of rehabilitation among workers with adjustment disorders according to practice guidelines: a retrospective cohort study. Occupational and Environmental Medicine, England, v. 60, Suppl. 1, p. 21-25, 2003.

NUNES, C. M. P.; ANDRADE, A. G. M. Terapia ocupacional e dor no campo das relações entre saúde e trabalho. In: DE CARLO, M. M. R. P.; QUEIROZ, M. E. G. (Org.). Dor e cuidados paliativos - terapia ocupacional e interdisciplinaridade. São Paulo: Rocca, 2007. p. 210-257.

OPSTEEGH, L. et al. Determinants of return to work in patients with hand disorders and hand injuries. Journal of Occupational Rehabilitation, Netherlands, v. 19, n. 3, p. 244-245, may 2009. Disponível em: < http:// www.springerlink.com/content/h36g57277168h141/ fulltext.pdf $>$. Acesso em: 20 maio 2009.

ORFALE, A. G. et al. Translation into Brazilian Portuguese, cultural adaptation and evaluation of the reliability of the disabilities of the arm, shoulder and hand questionnaire. Brazilian Journal of Medical and Biological Research, Brazil, v. 38, n. 2, p. 293-302, 2005.

PRANSKY, G. et al. Measuring functional outcomes in work-related upper extremity disorders. Development and validation of the Upper Extremity Function Scale, Journal of Occupational and Environmental Medicine, United States, v. 39, n. 12, p. 1195-1202, 1997.

SATO, L. LER: objeto e pretexto para a construção do campo trabalho e saúde. Cadernos de Saúde Pública, Rio de Janeiro, v. 17, n. 1, p. 147-152, jan./fev. 2001.

SATO, L. et al. Atividade em grupo com portadores de L.E.R. e achados sobre a dimensão psicossocial. Revista Brasileira de Saúde Ocupacional, São Paulo, v. 21, n. 79, p. 49-62, 1993.

SETTIMI, M. M. et al. Lesões por esforços repetitivos/ distúrbios osteomusculares relacionados ao trabalho: abordagem interdisciplinar. Revista do Projeto de Cooperação Técnica Brasil-Itália "Proteção à Saúde nos Ambientes de Trabalho”, p. 149-153, 1998.

SIQUEIRA, A. R.; QUEIROZ, M. F. F. Abordagem grupal em saúde do trabalhador. O mundo da saúde, São Paulo, v. 25, n. 4, p. 411-419, 2001.

SLUITER, J. K.; FRINGS-DRESEN, M. H. Quality of life and illness perception in working and sicklisted chronic RSI patients. International Archives of Occupational and Environmental Health, Germany, v. 81, n. 4, p. 495-501, july 2008.

TAKAHASHI, M. A. B. C.; CANESQUI, A. M. Pesquisa avaliativa em reabilitação profissional: a efetividade de um serviço em desconstrução. Cadernos de Saúde Pública, Rio de Janeiro, v. 19, n. 5 , p. 1473-1483, set./out. 2003.

VASSELJEN, J. R. O.; JOHANSEN, B. M.; WESTGAARD, R. H. The effect of pain reduction on perceived tension and EMG-recorded trapezius muscle activity in workers with shoulder and neck pain. Scandinavian Journal Rehabilitation Medicine, Sweden, v. 27, n. 4, p. 243-252, 1995.

YENG, L. T. Reabilitação em lesões por esforços repetitivos. In: CODO W. E.; ALMEIDA, M. C. C. G. (Org.). LER - Lesões por Esforços Repetitivos. Petrópolis: Vozes, 1995. p. 89-109.

YENG, L. T. et al. Distúrbios osteomusculares relacionados ao trabalho. In: TEIXEIRA, M. J. Dor, epidemiologia, fisiopatologia, avaliação, síndromes dolorosas e tratamento. São Paulo: Grupo Editorial Moreira Jr., 2001. p. 237-253.

ZIMERMAN, D. E. Fundamentos teóricos In: ZIMERMAN, D. E.; OSÓRIO, L. Como trabalhamos com grupos. Porto Alegre: Artes Médicas, 1997. p. 23-31. 